\title{
A 66-Year-Old Man With Fever and Altered Mental Status: A Case Report
}

\begin{abstract}
We describe the case of a 66-year-old man who was admitted to the Emergency Department with high fever, urinary incontinence, asthenia, and altered mental status characterized by drowsiness and lethargy from the previous night. The head CT scan was negative, while the chest $X$-ray showed a right basal reduced transparency and solitary pulmonary nodules already known and considered as expression of asbestosis. No acute neurological signs were observed by the consultant neurologist. A diagnosis of right basal pneumonia complicated by delirium was made and an empiric antibiotic therapy with ceftriaxone plus azithromycin was started. As patient's conditions did not improve in the following 48 hours, a lumbar puncture was performed, with the microbiological isolation of L. monocytogenes. According to the indications of the infectious disease consultant, a new antibiotic regimen with ampicillin/sulbactam plus gentamicin was introduced. The chest $C T$ scan performed as further examination revealed right pleural thickening highly suspicious for mesothelioma. The patient was discharged after 4 weeks with no neurological deficits.
\end{abstract}

Keywords: Altered Mental Status; Fever; Meningitis; Listeria monocytogenes

Un sessantaseienne con febbre e stato mentale alterato: un caso clinico

CMI 2018; 12(1): 37-42

https://doi.org/10.7175/cmi.v12i1.1350

\section{INTRODUCTION}

Differential diagnosis of fever is probably one of the most challenging topics in Internal Medicine, especially if not associated with specific symptoms or signs. Altered mental status can be related to the underlying infectious disease or be a symptom itself. A detailed investigation of patient's past medical history and risk factors, a complete physical examination and the execution of cultures before starting an antibiotic therapy are essential for the definition of diagnosis and treatment.

This case report allows to show the complexity of reaching the correct diagnosis and, consequently, the appropriate therapy.

\section{Why Do we Describe This Case}

We describe this case to point out how difficult could be to reach a correct diagnosis when signs and symptoms are nonspecific and could be referred to many different causes. Additionally, our patient had radiological sign of pneumonia which could explain the clinical presentation, but the absence, at the onset, of typical signs of lower respiratory tract infections could suggest a hematogenous pulmonary localization of Listeria. The alteration of his mental status was considered subsequent to age and fever

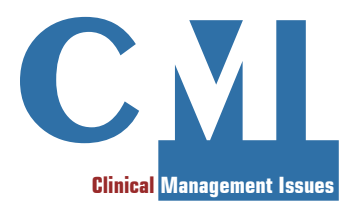

Case Report
Internal Medicine Unit, ASST Valle Olona,

Saronno Hospital, Italy
Corresponding author Paolo Ghiringhelli paolo.ghiringhelli@asst-valleolona.it

Received: 31 January 2018 Accepted: 15 May 2018 Published: 24 May 2018 


\begin{tabular}{|l|c|}
\hline \multirow{2}{*}{\multicolumn{1}{c|}{ Antihiotic }} & Listeria monocytogenes \\
\cline { 2 - 2 } Amoxicillin/clavulanate & $\mathrm{S} / \mathrm{B} / \mathrm{l}$ \\
Ampicillin & $\mathrm{S}$ \\
Cefotaxime & $\mathrm{S}$ \\
Chloramphenicol & $\mathrm{R}$ \\
Erythromycin & $\mathrm{R}$ \\
Moxifloxacin & $\mathrm{I}$ \\
Rifampicin & $\mathrm{R}$ \\
Tetracycline & $\mathrm{R}$ \\
Co-trimoxazole & $\mathrm{R}$ \\
\hline
\end{tabular}

Table I. Antimicrobial susceptibility of Listeria monocytogenes.

$\mathrm{I}=$ intermediate;

$\mathrm{MIC}=$ minimal inhibitory concentration;

$\mathrm{R}=$ resistant;

$\mathrm{S}=$ susceptible and Pneumococcal urinary antigen tests were performed and an intravenous empiric antibiotic therapy with ceftriaxone plus azithromycin was then started, as well as intravenous fluids.

Urine test and antigen test turned out all negative. Blood samples were analyzed also for Interferon Gamma Release Assays (in our hospital the IGRA Test is executed with the Quantiferon ${ }^{\circledR}$ TB Gold), which appeared to be positive; a chest $\mathrm{CT}$ scan with contrast was then scheduled.

Finally, the patient underwent to a head CT scan without contrast, which was negative for acute focal lesions, cerebral masses, or abscesses.

Since the patient's conditions did not improve in the following 48 hours, especially he was increasingly asthenic and lethargic and he answered to simple questions only if pressured, a new neurological consultation was requested, suspecting a Central Nervous System (CNS) involvement. A lumbar puncture was eventually carried out, showing a moderately turbid cerebrospinal fluid (CSF). The microscopic examination revealed 112 cells/ $\mu 1$ (normal values $0-5$ cells/ $\mu \mathrm{l}$ ) with prevalence of polymorphonuclear neutrophils and no bacterial cells; at the biochemical analysis, CSF glucose was reduced (13 $\mathrm{mg} / \mathrm{dl}$, with plasma glucose of $122 \mathrm{mg} / \mathrm{dl}$, with normal values among $60-70 \%$ of plasma glucose, i.e. $73-85 \mathrm{mg} / \mathrm{dl}$ ) while CSF proteins were increased $(129 \mathrm{mg} / \mathrm{dl}$, normal values $15-45 \mathrm{mg} / \mathrm{dl}$ ), suggesting a bacterial meningitis. Few hours later, a Gram positive bacillus highly suggestive for $L$. monocytogenes was identified in the CSF culture. Table I reports antimicrobial susceptibility of Listeria monocytogenes.

Antibiotic therapy was then shifted, according to the indications of the infectious disease consultant, from ceftriaxone plus azithromycin to ampicillin/sulbactam plus gentamicin; on the $4^{\text {th }}$ hospital day, also one of the three blood cultures became positive for L. monocytogenes, susceptible to penicillin. In few days the patient's clinical and neurological conditions improved and the fever disappeared. The antibiotic regimen was prescribed for 21 days.

Moreover, the chest CT scan organized to better define the pulmonary nodules showed right pleural thickening with focal enhancement and significant pleural effusion, as well as right pulmonary nodules with pathologic enhancement, confirmed by the following position emission tomography (PET) and suggestive for pleural mesothelioma. 
The patient was discharged after 4 weeks of hospitalization without neurological deficits.

After 1 month a video-assisted thoracoscopic surgery (VATS) for pleural biopsy and pleurodesis was performed. Histological examination revealed malignant cells compatible with primitive lung adenocarcinoma. Therefore, the patient was referred to an oncologist and a thoracic surgeon in order to define his further therapeutic plan.

\section{DISCUSSION}

L. monocytogenes is the most frequent human pathogen of Listeria genus, followed by L. ivnovii [1]. Listeria is a Gram positive, facultatively anaerobic, no spore-forming, motile, rod-shaped bacterium. It is a facultative intracellular bacillus, which can be isolated in soil, vegetation, wood, animals and decaying matter in the natural environment, thanks to its ability to survive in extreme conditions, as wide $\mathrm{pH}$ range, high salt concentrations, or at refrigeration temperatures [2].

Listeriosis is a bacterial food-borne infection, with a fatality rate of up to $30 \%$ when involves CNS, despite adequate antibiotic treatment [3]. Transmission occurs through the ingestion of contaminated food, especially undercooked food, raw vegetables or seafood, non-pasteurised cheese and milk [4,5]. It is responsible for the highest hospitalization and mortality amongst foodborne [5].

Proton pump inhibitors (which our patient was taking due to his previous history of duodenal ulcer) and other drugs reducing gastric acid increase the risk of illness, because gastric low $\mathrm{pH}$ represents an important barrier to Listeria [6].

Its incidence in Europe is $0.34-0.52$ per 100,000 inhabitants per year [7], and it mainly occurs in particular at-risk groups: pregnant women, elderly (considered as aged over 50 years old), immunosuppressed transplant recipients, patients with impaired cell-mediated immunity, diabetics, and neonates. Malignancy is the main predisposing risk factor [8].

In immunocompetent people, L. monocytogenes might cause febrile gastroenteritis, which is usually mild and self-limiting in few days, after a incubation period variable from 24 hours to 3 weeks. In at-risk groups, this bacterium is an important cause of life-threatening infections including sepsis, meningitis, or meningoencephalitis and less commonly rhomboencephalitis, due to its tropism to the central nervous system [9]. Less often, brain abscesses due to Listeria have been reported [10].

L. monocytogenes is the fourth most frequent cause of bacterial meningitis [11]. The typical triad of fever, neck stiffness, and altered mental status is described only in $44 \%$ of patients with meningitis, but $95 \%$ has at least two of the four symptoms among headache, fever, neck stiffness, and altered mental status [12]. However, the incidence of meningeal signs among patients with $L$. monocytogenes is lower than that among cases of meningitis due to other causes of bacterial meningitis [13].

Patients with L. monocytogenes meningitis are frequently comatose and they do not develop a rapid progression to respiratory failure, suggesting that the disease is not as rapidly progressive as pneumococcal meningitis [14].

In elderly people, clinical presentation could be troublesome, as main clinical symptoms such as fever or neck stiffness may be absent [15] or related to other diseases typical of aging patients, as Parkinson's disease or cervical arthritis; an altered mental status could be expression of several infectious or non-infectious underlying causes [16]. Moreover, its incidence approaches to $9 \%$ in this group [17].

Our patient did not present headache nor neck stiffness and denied dubiously preserved food ingestion; moreover, the chest X-ray showed a soft right basal opacity consistent with early radiological sign of pneumonia, which could explain the patient's symptoms. Antibiotic therapy for community-acquired pneumonia (CAP) was thus started.

As the symptoms did not improve, actually the level of consciousness worsened, a CNS involvement was suspected and the CSF examination showed pleocytosis associated with an increase in protein concentration and low CSF glucose as compared to the blood concentration, which are the typical findings of bacterial meningitis [12]. CSF cultures yielded L. monocytogenes susceptible to ampicillin and amoxicillin/clavulanate, resistant to cefotaxime, chloramphenicol, moxifloxacin, rifampicin, and tetracycline, and intermediate to erythromycin and cotrimoxazole (Table I). Susceptibility to gentamicin was not reported. 
Real-time polymerase chain reaction (PCR) assay is a relatively new and rapid test to detect $L$. monocytogenes, especially helpful when patients have received antibiotic therapy before the lumbar puncture execution, with a moderate sensitivity and specificity of 80\% [18]. We did not perform PCR analysis because, unfortunately, it is not available in our hospital. However, PCR assays must not replace $\mathrm{CSF}$ and blood cultures, as these are the only techniques which test antimicrobial susceptibility.

According to guidelines [19,20], the treatment of choice of $L$. monocytogenes meningitis is ampicillin plus a synergistic aminoglycoside for at least 3 weeks. In patients with $\beta$-lactams allergy, co-trimoxazole is the best alternative thanks to its favorable penetration through the blood-brain barrier [21]. Fluoroquinolones accumulate in monocytes, which are the target cells of $L$. monocytogenes, and are rapidly bactericidal; however, their clinical activity is not as high as in experimental models, apparently because these antibiotics are less active against intracellular than extracellular forms of $L$. monocytogenes [22]. Linezolid has a proven in vitro activity against this bacterium, but clinical data are currently limited [23]. Meropenem has reported to cause a higher mortality compared to penicillin, in spite of its low minimum inhibitory concentration (even lower than that of ampicillin) against L. monocytogenes, supposedly due to some difference in their intracellular activity [24]. Vancomycin is bactericidal within six hours, but it is not able to cross the blood-brain barrier reaching therapeutic concentration, thus its use is limited in cases of meningitis [25]. Cephalosporins are ineffective while steroids are not recommended [26].

We initially considered pneumonia as consequent to the systemic dissemination of $L$. monocytogenes; then, after the improvement of the patient's conditions, we performed a chest CT and a whole body PET scan which suggested the likely neoplastic nature of the pulmonary nodules, subsequently confirmed by histological examination, attesting malignancy as the main risk factor for L. monocytogenes meningitis.

\section{CONCLUSION}

This case emphasizes the need of considering $L$. monocytogenes meningitis in the differential diagnosis of fever and altered mental status in any patient, even if immunocompetent, especially in the elderly, due to its high mortality rate.

Moreover, with the increasing number of the elderly population and the spread of vaccinations against $H$. influenzae, $S$. pneumoniae, and N. meningitis, L. monocytogenes meningitis is expected to become more frequent in adult population.

Finally, when a L. monocytogenes meningitis is diagnosed in an immunocompetent patient, a detailed investigation of risk factors should be done to rule out underlying neoplastic diseases.

The most common errors

- Not investigating the past medical history

- Not performing complete physical examination

- Not considering disturbance of consciousness as expression of an underlying neurological disease but only age-related or fever-related; it is always necessary to ask about the previous mental status

- Delaying blood cultures and lumbar puncture

- Delaying antibiotic prescription

\section{Funding}

This article has been published without the support of sponsors.

Conflicts of interests

Authors declare they have no competing financial interests concerning the topic of this article. 


\section{REFERENCES}

1. Guillet C, Join-Lambert O, Le Monnier A, et al. Human listeriosis caused by Listeria ivanovii. Emerg Infect Dis 2010; 16: 136-8; https://doi.org/10.3201/eid1601.091155

2. Vázquez-Boland JA, Kuhn M, Berche P, et al. Listeria pathogenesis and molecular virulence determinants. Clin Microbiol Rev 2001; 14: 584-640; https://doi.org/10.1128/CMR.14.3.584640.2001

3. Arslan F, Meynet E, Sunbul M, et al. The clinical features, diagnosis, treatment, and prognosis of neuroinvasive listeriosis: a multinational study. Eur J Clin Microbiol Infect Dis 2015; 34: 121321; https://doi.org/10.1007/s10096-015-2346-5

4. Ooi ST, Lorber B. Gastroenteritis due to Listeria monocytogenes. Clin Infect Dis 2005; 40: 1327-32; https://doi.org/10.1086/429324

5. Barton Behravesh C,Jones TF, Vugia DJ, et al; FoodNet Working Group. Deaths associated with bacterial pathogens transmitted commonly through food: foodborne diseases active surveillance network (FoodNet), 1996-2005.J Infect Dis 2011; 204: 263-7; https://doi.org/10.1093/infdis/ jir263

6. Bavishi C, Dupont HL. Systematic review: the use of proton pump inhibitors and increased susceptibility to enteric infection. Aliment Pharmacol Ther 2011; 34: 1269-81; https://doi. org/10.1111/j.1365-2036.2011.04874.x

7. Zolin A, Amato E, D'Auria M, et al. Estimating the real incidence of invasive listeriosis through an integrated surveillance model in use in Lombardy (Italy, 2006-2014). Epidemiol Infect 2017; 145: 2072-80; https://doi.org/10.1017/S0950268817000711

8. de Noordhout CM, Devleesschauwer B, Angulo FJ, et al. The global burden of listeriosis: a systematic review and meta-analysis. Lancet Infect Dis 2014; 14: 1073-82; https://doi. org/10.1016/S1473-3099(14)70870-9

9. Doganay M. Listeriosis: clinical presentation. FEMS Immunol Med Microbiol 2003; 35: 173-5; https://doi.org/10.1016/S0928-8244(02)00467-4

10. Bartt R. Listeria and atypical presentations of Listeria in the central nervous system. Semin Neurol 2000; 20: 361-73; https://doi.org/10.1055/s-2000-9398

11. Thigpen MC, Whitney CG, Messonnier NE, et al; Emerging Infections Programs Network. Bacterial meningitis in the United States, 1998-2007. NEnglJ Med 2011; 364: 2016-25; https:// doi.org/10.1056/NEJMoa1005384

12. van de Beek D, de Gans J, Spanjaard L, et al. Clinical features and prognostic factors in adults with bacterial meningitis. N Engl J Med 2004; 351: 1849-59. Erratum in: N Engl J Med 2005; 352: 950. https://doi.org/10.1056/NEJMoa040845

13. Mylonakis E, Hohmann EL, Calderwood SB. Central nervous system infection with Listeria monocytogenes. 33 years' experience at a general hospital and review of 776 episodes from the literature. Medicine (Baltimore) 1998; 77: 313-36; https://doi.org/10.1097/00005792199809000-00002

14. Pagliano P, Attanasio V, Rossi M, et al. Listeria monocytogenes meningitis in the elderly: distintive characteristics of the clinical and laboratory presentation. J Infect 2015; 71: 134-6; https://doi.org/10.1016/j.jinf.2015.02.003

15. High KP, Bradley SF, Gravenstein S, et al; Infectious Diseases Society of America. Clinical practice guideline for the evaluation of fever and infection in older adult residents of longterm care facilities: 2008 update by the Infectious Diseases Society of America. J Am Geriatr Soc 2009; 57: 375-94; https://doi.org/10.1111/j.1532-5415.2009.02175.x

16. Magazzini S, Nazerian P, Vanni S, et al. Clinical picture of meningitis in the adult patient and its relationship with age. Intern Emerg Med 2012; 7: 359-64; https://doi.org/10.1007/s11739012-0765-1

17. Pagliano P, Ascione T, Boccia G, et al. Listeria monocytogenes meningitis in the elderly: epidemiological, clinical and therapeutic findings. Infez Med 2016; 24: 105-11

18. Jaton K, Sahli R, Bille J. Development of polymerase chain reaction assays for detection of Listeria monocytogenes in clinical cerebrospinal fluid samples. JClin Microbiol 1992; 30:1931-6

19. Hof H, Nichterlein T, Kretschmar M. Management of listeriosis. Clin Microbiol Rev 1997; 10: $345-57$

20. Tunkel AR, Hartman BJ, Kaplan SL, et al. Practice guidelines for the management of bacterial meningitis. Clin Infect Dis 2004; 39: 1267-84; https://doi.org/10.1086/425368

21. Merle-Melet M, Dossou-Gbete L, Maurer P, et al. Is amoxicillin-cotrimoxazole the most appropriate antibiotic regimen for listeria meningoencephalitis? Review of 22 cases and the literature. J Infect 1996; 33: 79-85; https://doi.org/10.1016/S0163-4453(96)92929-1 
22. van de Velde S, Nguyen HA, van Bambeke F, et al. Contrasting effects of human THP-1 cell differentiation on levofloxacin and moxifloxacin intracellular accumulation and activity against Staphylococcus aureus and Listeria monocytogenes. J Antimicrob Chemother 2008; 62: 518-21; https://doi.org/10.1093/jac/dkn232

23. Callapina M, Kretschmar M, Dietz A, et al. Systemic and intracerebral infections of mice with Listeria monocytogenes successfully treated with linezolid.J Chemother 2001; 13:265-9; https:// doi.org/10.1179/joc.2001.13.3.265

24. Thønnings S, Knudsen JD, Schønheyder HC, et al. Antibiotic treatment and mortality in patients with Listeria monocytogenes meningitis or bacteraemia. Clin Microbiol Infect 2016; 22: 725-30; https://doi.org/10.1016/j.cmi.2016.06.006

25. Arsene O, Linassier C, Quentin R, et al. Development of listeriosis during vancomycin therapy in a neutropenic patient. Scand J Infect Dis 1996; 28: 415-6; https://doi. org/10.3109/00365549609037930

26. Heyderman RS; British Infection Society. Early management of suspected bacterial meningitis and meningococcal septicaemia in immunocompetent adults--second edition.J Infect 2005; 50: 373-4; https://doi.org/10.1016/j.jinf.2005.04.002 